\title{
Belphégor
}

\section{La littérature dans les feuilletons des journaux viennois dans l'année 1855}

\section{Norbert Bachleitner}

\section{(e) OpenEdition}

1 Journals

\section{Electronic version}

URL: https://journals.openedition.org/belphegor/2354

DOI: 10.4000/belphegor.2354

ISSN: 1499-7185

Publisher

LPCM

\section{Electronic reference}

Norbert Bachleitner, "La littérature dans les feuilletons des journaux viennois dans l'année 1855", Belphégor [Online], 18-1 | 2020, Online since 07 February 2020, connection on 30 June 2021. URL: http://journals.openedition.org/belphegor/2354 ; DOI: https://doi.org/10.4000/belphegor.2354

This text was automatically generated on 30 June 2021.

\section{(c) (i) (9)}

Belphégor est mis à disposition selon les termes de la Licence Creative Commons Attribution - Pas d'Utilisation Commerciale - Pas de Modification 4.0 International. 


\title{
La littérature dans les feuilletons des journaux viennois dans l'année 1855
}

\author{
Norbert Bachleitner
}

Comparée à l'Europe de l'Ouest, la presse populaire s'établit seulement avec un certain retard dans la monarchie habsbourgeoise. Le processus de modernisation de la société, notamment l'urbanisation, l'expansion de l'éducation, la participation de la population aux décisions politiques avec un système d'information, de communication et discussion publique, ${ }^{1}$ ne commencent à se former qu'après la révolution de 1848 . C'est donc l'époque dite néo-absolutiste (1848-1860), une époque de restauration mais en même temps de modernisation modérée, qui voit paraître à Vienne les premiers journaux populaires. Plus que les autres journaux, la presse populaire est dès le début un mélange d'information et de divertissement, et inclut également le romanfeuilleton. Dans ce type de presse le roman ne sert pas seulement à la récréation mais aussi à la construction d'un espace mental collectif pour la population. Le romanfeuilleton populaire, prototype de la littérature hétéronome selon la terminologie proposée par Pierre Bourdieu, ${ }^{2}$ est donc beaucoup plus intégré dans l'ensemble du journal que le feuilleton littéraire dans la presse dite de « qualité » où l'on trouve de la littérature autonome.

Comparée avec les autres villes de la monarchie habsbourgeoise, dans les années 1850 la diversification de la presse a été la plus avancée à Vienne. On y peut distinguer au moins trois groupes de journaux qui se différencient clairement par le tirage et le prix. Il s'agit de la presse de "qualité », de la presse populaire et d'une petite couche intermédiaire qu'on pourrait appeler la presse moyenne. La presse de « qualité » avait un tirage de 1400 à 5200 exemplaires et un prix entre 10 et 20 livres par année. On y trouve les feuilles suivantes: ${ }^{3}$

Wiener Zeitung (A. 5250, Jg. 20 fl.)

Österreichische Zeitung (A. 5000, Jg. 12 fl.)

Der Wanderer (A. 5000, Jg. 12 fl.) 
Ostdeutsche Post (A. 4000, Jg. 12 fl.)

Donau (A. 2600, Jg. 10 fl.)

Der Humorist (A. 1400, Jg. 12)

Dans le secteur moyen on trouve la Presse avec un prix annuel de 8 livres et un tirage de 17.000 exemplaires et le Fremdenblatt avec un prix de l'abonnement de 6 livres et un tirage de 12.500 exemplaires. Les représentants de la presse populaire sont les suivants:

Morgen-Post (A. 19.000, Jg. 4 3/4 fl.)

Stadt- und Vorstadt-Zeitung (A. 8000, Jg. 4 3/4 fl.)

Wiener Telegraf(A. 6000, Jg. 4 fl.)

Wiener Neuigkeits-Blatt (A. 2300, Jg. 4 fl.)

Wiener Kourier (A. 1300, Jg. 4 fl.)

Les journaux populaires se vendent au numéro, dont le prix se chiffre entre un et trois kreuzer, l'équivalent du sou français. La popularité de quelques journaux populaires est garantie par leurs éditeurs, tels Julius Seidlitz, fondateur du Stadt- und Vorstadt-Zeitung, et Adolf Bäuerle, rédacteur en chef du Wiener Telegraf, tous les deux protagonistes importants de la littérature populaire viennoise. Les maisons d'édition Stöckholzer von Hirschfeld (Morgen-Post, Wiener Telegraf) et Jasper's Witwe \& Hügel, elles aussi, sont liées au roman-feuilleton par leur rôle d'éditeurs de littérature «facile ». Les journaux populaires se consacrent au reportage des événements locaux et des faits divers; s'ils poursuivent une tendance politique, c'est une tendance "démocrate " et de porteparole du peuple. En témoignent maints commentaires du côté de la justice en forme de saisies et amendes, de l'Eglise et de la critique littéraire. Le roman-feuilleton corromprait la morale des classes sociales inférieures par ses portraits défigurant les couches sociales supérieures, il insulterait des personnages vivants insérés dans les romans comme caractères à clé, il minimiserait les dangers de la criminalité - bref : il serait nuisible à l'individu et à la société.

Sans aucun doute le public des journaux de «qualité » était composé surtout par des lecteurs aisés et bien éduqués alors que les journaux populaires étaient lus par la petite bourgeoisie et les couches inférieures. ${ }^{5}$ Lorsque l'on fait référence aux «travailleurs » ou même aux " prolétaires » dans les journaux populaires c'est à l'entière population active, y compris les artisans, les petits commerçants et les employés modestes que les contemporains pensaient. Si l'on considère les sujets des articles dans la presse populaire il est clair qu'ils visent exactement une telle clientèle.

En 1857 fut effectué un recensement de la population autrichienne qui, pour la première fois, enregistra la profession. ${ }^{6}$ Nous essayerons, dans les lignes suivantes, de montrer la correspondance entre les tirages des deux groupes de journaux mentionnés ci-dessus et la répartition sociale de la population. On rassemblera donc, d'un côté, l'aristocratie et la haute bourgeoisie et de l'autre la petite bourgeoisie et les travailleurs.

\begin{tabular}{|l|l|}
\hline La population viennoise en 1857 & \\
\hline Total : 514087 & \\
dont & \\
\hline
\end{tabular}




\begin{tabular}{|c|c|c|}
\hline \multicolumn{2}{|l|}{$\begin{array}{l}\text { Autochtones : } 284999 \\
\text { dont }\end{array}$} & \multirow[t]{2}{*}{ Étrangers : 229088} \\
\hline Clergé & 890 & \\
\hline Fonctionnaires & 9207 & \\
\hline Militaires & 774 & \\
\hline Auteurs, artistes & 2995 & \\
\hline Avocats, notaires & 376 & \\
\hline Services de santé & 1138 & \\
\hline Propriétaires fonciers et de mines, fermiers & 253 & \\
\hline Propriétaires de maison, rentiers & 5490 & \\
\hline Fabricants, artisans & 24151 & \\
\hline Commerçants & 3668 & \\
\hline Bateliers, pêcheurs & 185 & \\
\hline Manœuvres dans l'agriculture, mineurs & 381 & \\
\hline Manœuvres divers & 27729 & \\
\hline Manœuvres dans le commerce & 3308 & \\
\hline Domestiques & 10417 & \\
\hline Journaliers & 7258 & \\
\hline Hommes de plus de 14 ans & 7920 & \\
\hline Femmes et enfants & 178859 & \\
\hline Total & 284999 & \\
\hline
\end{tabular}

Les fabricants, artisans et commerçants devront être répartis dans un quart de bourgeoisie aisée (6.950) et trois quarts de petite bourgeoisie (20.850). ${ }^{7}$ De même, le groupe de fonctionnaires se compose d'un quart de membres des couches supérieures (2.300) et de trois quarts de petite bourgeoisie (6.900). ${ }^{8}$ Par contre, on compte trois quarts du clergé, des militaires, des auteurs et des artistes parmi les couches supérieures (4.620), un quart parmi la petite bourgeoisie (1.540). Finalement, on peut compter les propriétaires fonciers et de maisons ainsi que les rentiers parmi l'aristocratie et la bourgeoisie aisée (5.740). Autrement dit, en chiffres arrondis, les classes élevées viennoises comptent 19.600 personnes, la petite bourgeoisie 29.300 
personnes, et les couches inférieures (travailleurs) 49.200 personnes (sans femmes et enfants).

Sans doute un modeste pourcentage des travailleurs lisait aussi des journaux populaires. Il reste les "étrangers", dont la plupart sont des artisans et travailleurs immigrés des provinces de la monarchie, notamment de la Bohème, de la Moravie et de la Silésie. ${ }^{9}$ On doit donc ajouter un certain nombre des 229.000 étrangers au lectorat des journaux populaires.

Malgré une inévitable part d'imprécision, on peut constater une certaine correspondance entre les chiffres de la population et ceux des tirages de la presse. Les journaux de «qualité » en langue allemande ont un tirage de 23.250 exemplaires, les journaux populaires un tirage de 36.600 . Les feuilles du champ intermédiaire comptent 29.500 exemplaires; il faut probablement les partager entre les couches supérieures et la petite bourgeoisie. D'après ce calcul nous arrivons à un total d'environ 38.000 exemplaires de feuilles pour le lectorat élevé et 52.000 exemplaires pour le "peuple ». Il faut aussi prendre en considération qu'à peu près la moitié des exemplaires de feuilles de " qualité » sont destinés à des provinces de la monarchie ou même à l'extérieur. Il va sans dire que la réalité de la lecture des journaux était plus complexe avec des lecteurs des couches élevées feuilletant les journaux populaires et une partie du lectorat $d u$ «peuple» lisant les feuilles de "qualité » et «moyennes ». Il s'agit ici seulement de montrer une homologie entre la stratification sociale et l'offre de lecture périodique.

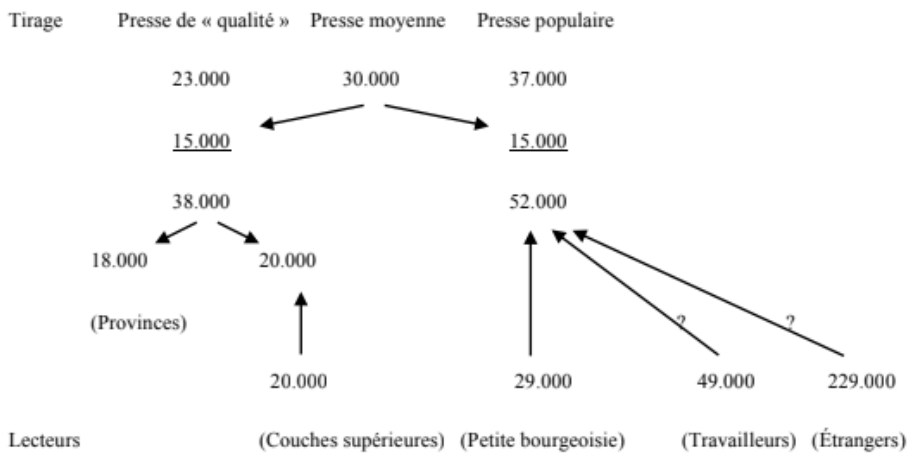

Il serait sans doute exagéré de parler d'un champ de la presse, en empruntant le terme de Bourdieu, dans la monarchie habsbourgeoise des années $1850,{ }^{10}$ puisqu'il manquait encore l'autonomie de la presse et le libre jeu des forces et des agents. Tout de même on observe déjà une tendance importante dans cette direction. Les journaux commencent à se distinguer l'un de l'autre, et cela aussi par la littérature qu'ils accueillent dans leur feuilleton.

Cette structuration d'un champ primitif se traduit non seulement par une distinction entre des journaux élitistes et des journaux populaires, mais aussi par une différenciation à l'intérieur des groupes de journaux. D'ailleurs, quelques-uns d'entre eux dépendaient du gouvernement, alors que d'autres faisaient de l'opposition prudente. 
La littérature, elle aussi, se divise en un secteur autonome et un secteur hétéronome. Depuis le romantisme, la littérature cherche l'autonomie des influences externes, y compris le goût du public. La presse de «qualité » donne la préférence au genre de la "nouvelle» (d'une ambivalence sémantique remarquable) et pas au roman. La littérature dans ce secteur de la presse se refuse à chaque commentaire direct de la vie quotidienne. Le Donau, exemple de la presse de "qualité » fondé en 1854, contient maints articles sur des questions économiques. Dans le domaine de la littérature le journal essaie de rendre compte des nouveautés en librairie et au théâtre, il s'occupe d'auteurs classiques tels que Goethe, Wieland, Pouchkine, Lermontov ou Petőfi, donc de la littérature dite « légitime ».

Par contre, la littérature dans les journaux populaires est l'incarnation de l'hétéronomie, c'est-à-dire d'un art utile dans lequel des phénomènes extra-littéraires (politiques, religieux, didactiques, économiques...) exercent une domination sur les aspects artistiques. Le contact direct avec le lectorat petit bourgeois qui a un fort lien avec ses alentours immédiats ${ }^{11}$ donne au roman local un important degré d'hétéronomie qui le place près de la communication orale. Le Wiener Kourier publie des nouvelles internationales, des articles sur des questions sociales concernant le peuple, par exemple sur les raisons de la hausse des prix («Die Ursachen der herrschenden Theuerung "; Nr. 38, 42), il publie aussi des faits divers (La fiancée sans robe de mariée ; Les jumeaux siamois africains; Nr. 21: «Die Braut ohne Brautkleid», Nr. 24: «Die afrikanischen zusammengewachsenen Zwillinge »), et des illustrations sensationnalistes comme celle de la guerre de Crimée (Nr. 25: « Ajax, der französische Heldenhund, auf dem Schlachtfelde an der Tschernaja "; voir l'illustration ci-dessous). Le feuilleton comporte un mélange de contes historiques, anecdotes et romans, dont Die Wiener Kartenaufschlägerin und die Modistin de Betty Ortwein v. Molitor, issu d'un concours ouvert par le journal.

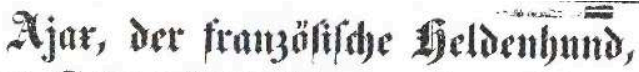

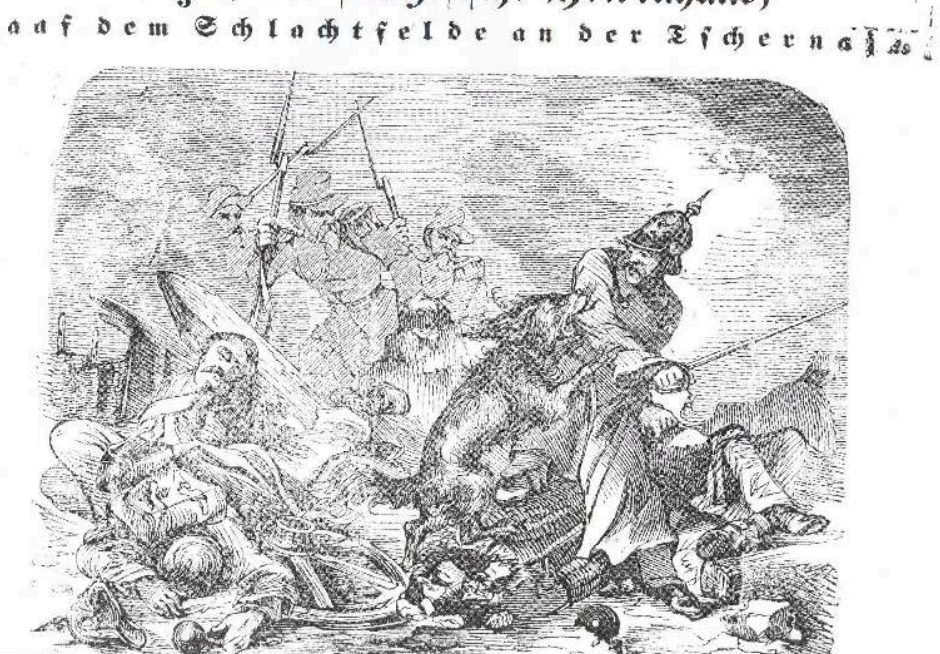

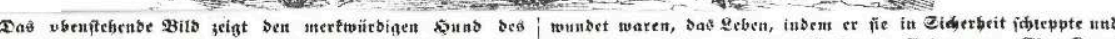

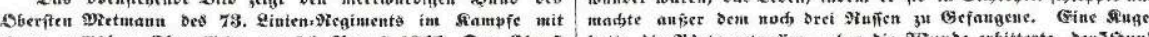

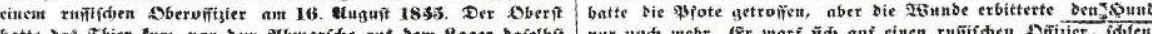

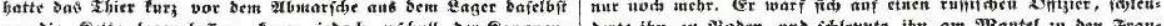

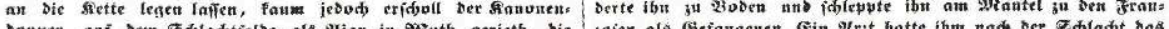

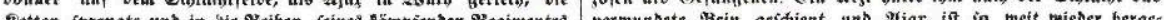

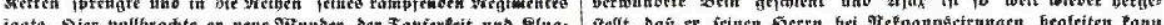
beit. elo vour

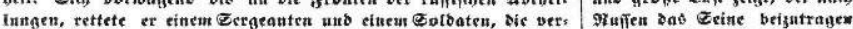

La littérature dans la presse de «qualité » se distingue de la presse populaire par la quantité de capital symbolique accumulée par les auteurs et les genres choisis 
(nouvelles et autres formes de prose courte de préférence au roman), ${ }^{12}$ style et thématique (introspection, réflexion, " poésie " plutôt que réalisme et aventures) ${ }^{13} \mathrm{le}$ degré d'intégration dans le journal (reproduction après publication en librairie dans un cas, roman écrit pour le feuilleton, dans l'autre), ${ }^{14}$ et la provenance (littérature étrangère, voire mondiale plutôt que littérature locale). ${ }^{15}$

Quant à la littérature étrangère, il faut rappeler le modèle du transfert international proposé par Pascale Casanova. Selon elle, les pays avec une littérature peu développée préfèrent recevoir des œuvres qui se placent autour du pôle autonome du champ littéraire afin de renforcer la littérature légitime du pays accueillant. C'est exactement ce qui se passe dans les journaux de "qualité » autrichiens qui s'orientent vers la littérature légitime européenne. Par contre, la littérature au pôle hétéronome du champ littéraire est destinée à des fins nationales et politiques. ${ }^{16} \mathrm{Il}$ est donc tout à fait logique que les journaux populaires et locaux fassent confiance à la littérature du pays.

\section{Autonomie vs. Hétéronomie: Das Goldmärchen (dans Donau) et Die Wiener Kartenaufschlägerin und die Modistin (dans Wiener Kourier)}

L'auteur de Das Goldmärchen, Ferdinand Kürnberger, choisit pour son récit allégorique le genre littéraire du conte de fée, genre légitime à l'époque du romantisme. Comme dans quelques autres de ses œuvres, dans ce récit l'argent est la raison de l'échec des relations humaines (« Urheber des Scheiterns menschlicher Beziehungen »). ${ }^{17}$ Emma, une aristocrate, aime Heldwin, le fils d'un commerçant qui travaille comme paysan près de son château. Mais elle succombe à la tentation de la richesse quand elle rencontre un comte qui est en route vers le "pays d'or " découvert récemment par Columbus. Au pays d'or règne un ogre nommé Oog; s'enfuyant de lui les voyageurs se rendent aux " îles de la confiance" (Inseln des guten Vertrauens) où les plumes de cygnes font fonction d'argent. Les détenteurs des plumes espèrent que celles-ci seront un jour changées en or. Quand ils discernent que cette espérance est fausse, une révolution éclate. Emma se rend à "l'île des mains de fer " (Insel der eisernen Hände), où la population pour se nourrir doit exercer du travail manuel dur. Désillusionnée, Emma retourne à son pays et épouse le fils du commerçant. Elle professe désormais la foi non en l'or et l'aristocratie mais dans le travail des champs. ${ }^{18}$

Même si le récit est assez simple, il serait abusif de le réduire à un message antimonétaire. Certains motifs secondaires cherchent à engendrer de la " poésie », les lieux allégoriques donnent un sens beaucoup plus vaste au récit que l'opposition entre argent, morale et émotion. Dans sa préface, l'auteur souligne que ses personnages suivent une logique de liberté et d'autonomie esthétiques (der « ästhetischen Freiheit und Selbständigkeit ») plutôt qu'une logique de morale pédagogique. Il insiste sur le fait qu'il y a un fluide inconcevable dans son récit (" ein unfaßbares Fluidum »). ${ }^{19}$

L'auteur lui-même revendique donc l'autonomie esthétique pour son récit. Un sujet banal tel que l'argent exige de la sublimation par l'esprit poétique. En s'appuyant sur la pensée de Bourdieu on peut ajouter que c'est seulement enveloppé dans un voile poétique qu'un tel sujet sera appréciée du goût bourgeois. ${ }^{20}$ On pourrait ici faire référence à la biographie de l'auteur qui, après l'échec de la révolution de 1848, avait décidé de retourner à la simple littérature (« zur bloßen Literatur »). ${ }^{21}$ Il essayait donc 
de se faire accepter par le milieu des auteurs consacrés, par exemple il espérait placer ses pièces au Burgtheater. Ajoutons que l'habitus de Kürnberger et l'effet de celui-ci sur son récit est caractéristique de la littérature préférée par les journaux dits de « qualité ».

En revanche, dans le roman de Betty Ortwein, notre exemple de roman publié dans la presse populaire, tout tourne autour de l'argent et de son influence désastreuse. L'incarnation de son effet pernicieux est la prophétesse dite "sorcière de Nikolsdorf ", qui fait l'entremetteuse entre les travailleuses et des hommes riches. Dans ce métier elle a accumulé un trésor de 40.000 livres. Sa victime est Johanna (Jeannette), une jeune modiste qui est pourchassée par un baron. Thérèse, son ancienne maîtresse, l'aide à faire chasser Johanna de son poste de travail. Thérèse insinue à l'oreille de sa patronne que Johanna aime son époux. Elle réussit aussi à rendre jaloux Anton, le fiancé de Johanna, qui par conséquent bat sa petite amie. Pour vérifier son soupçon il fait irruption chez le baron qui le fait arrêter comme voleur. Le baron éprouve du remords, il fait un don important à Johanna et son fiancé, se dénonce auprès de la justice et est mis en prison. La sorcière, elle aussi, est emprisonnée, tout semble tourner vers une fin agréable, quand Johanna avoue à Anton qu'elle aime le gentil baron. Il s'ensuit qu'Anton la tue.

A l'encontre du conte de fée de Kürnberger, les personnages dans le roman d'Ortwein peuvent être proches du lectorat des journaux populaires. Le lieu est la banlieue viennoise, l'apparence d'authenticité et de réalisme est créée par plusieurs références aux faits divers locaux.

Ortwein présente - par son roman - aux lectrices auxquelles elle s'adresse explicitement, des normes de comportement de la petite bourgeoisie, notamment la modération, avoir un caractère laborieux, l'anti-alcoolisme, la fidélité conjugale, la solidarité avec les membres de sa famille et de sa classe, et surtout l'idée de se contenter de sa modeste position dans la société. A la moitié du XIXè siècle nous pouvons parler d'un processus de formation de classe («Prozeß der Klassenbildung ») ${ }^{22}$ parmi les artisans et les petits commerçants autrichiens, y compris nombre de travailleurs immigrés de la périphérie de la monarchie habsbourgeoise.

La vertu symbolique qui caractérise et unifie la classe assez hétérogène de la petite bourgeoisie est l'honneur. Le respect du code d'honneur donne une sorte de capital symbolique, un certain pouvoir, qui peut garantir du crédit ou un mariage modeste mais respectable. La réputation intacte peut consoler du manque de biens matériels et notamment de capital économique. Une faute contre le code d'honneur est sanctionnée par l'exclusion immédiate, ce qui est démontré dans le roman d'Ortwein par les femmes qui succombent à la tentation du « péché » et/ou de l'argent.

Cette logique un peu paradoxale n'est pas facile à communiquer, il fallait donc la répéter constamment pour la faire entrer dans la tête des membres du groupe concerné. Dans le roman d'Ortwein, mais aussi dans nombre d'autres romans populaires, il y a maints exemples en faveur de la confirmation des avantages d'une vie vertueuse. Une fille incarcérée par la sorcière se défend contre les avances d'un chef d'usine et préfère rester travailleuse, une autre, tombée dans la misère, regrette de ne pas avoir choisi le droit chemin, et même le baron converti professe une morale petite bourgeoise.

Sauf le baron, tous les personnages viennent du peuple. Le roman met en scène des carrières tournant vers la catastrophe parce que les protagonistes, motivés par la soif 
d'argent, cherchent le contact avec les classes supérieures. Mais le code d'honneur est despotique, il ne permet pas une telle fuite. Adhérer à une classe et sauvegarder la normalité définie par le code a pour conséquence de pouvoir profiter de la solidarité, mais en même temps d'être exposé à la menace de sanctions sévères en cas de déviation. La tentation de négliger les normes de la petite bourgeoisie s'accroit à partir de 1850 à cause de la chute de l'artisanat due à la concurrence des usines. ${ }^{23}$ Nombre d'artisans devaient désormais travailler pour une grande entreprise (Verlagssystem), ainsi le paupérisme se répand, les travailleurs du nouveau type ne pouvaient plus se marier, donc le nombre d'enfants illégitimes augmente. Tous ces développements mènent à la prolétarisation d'une partie du peuple.

Quand on parle de la propagande morale par le roman populaire, il ne faut pas oublier que le roman à sensation local permet une double lecture : il peut consoler les garçons et les filles gentils et modestes, mais il permet aussi de jouir de la transgression des normes dans la fiction littéraire, on peut donc jouir de l'interdit dans le cadre du permis. Ainsi le roman populaire montre sa figure de Janus : il est à la fois porteur de normes morales, donc instrument d'édification, et médium de divertissement et de distraction.

\section{NOTES}

1. Cf. Gabriele Melischek, Josef Seethaler: Von der Lokalzeitung zur Massenpresse. Zur Entwicklung der Tagespresse im österreichischen Teil der Habsburgermonarchie nach 1848. Jahrbuch für Kommunikationsgeschichte 7 (2005), p. 52-92.

2. Voir Pierre Bourdieu: Les règles de l'art. Genèse et structure du champ littéraire. Paris: Seuil 1992.

3. On ne s'intéresse ci-après qu'aux journaux de langue allemande. A. = Auflage (tirage); Jg. = Jahrgang (prix de l'abonnement par année); fl. = Gulden (livres).

4. Chiffres selon Constant Wurzbach von Tannenberg: Bibliographisch-statistische Übersicht der Literatur des östreichischen Kaiserstaates vom 1. Jänner bis 31. December 1855. Dritter Bericht. Wien: k. k. Hof- und Staatsdruckerei 1857, vol. 1.

5. Cf. Karl-Heinz Kossdorff, Die Wiener Lokalpresse im 19. Jahrhundert. Von der Gründung des ersten Volksblattes bis zur Aufhebung des Zeitungsstempels (1850-1900). Dissertation, Universität Wien 1969, p. 108.

6. Tafeln zur Statistik der österreichischen Monarchie, N. F. Bd. 3. Wien: k. k. Hof- und Staatsdruckerei 1861.

7. Voir Ernst Bruckmüller: Wiener Bürger. Selbstverständnis und Kultur des Wiener Bürgertums vom Vormärz bis zum fin de siècle. In: Hannes Stekl, Peter Urbanitsch, Ernst Bruckmüller, Hans Heiss (Hg.), «Durch Arbeit, Besitz, Wissen und Gerechtigkeit». (Bürgertum in der Habsburgermonarchie 2) Wien, Köln, Weimar: Böhlau 1992, p. 43-68, p. 52.

8. Voir Gustav Adolf Schimmer, Die Bevölkerung von Wien und seiner Umgebung nach dem Berufe und der Beschäftigung. Wien: Ueberreuter 1874, première partie, p. 38.

9. Voir Ernst Bruckmüller, Sozialgeschichte Österreichs. Wien, München: Herold 1985, p. 388.

10. La théorie du champ littéraire est développée dans Pierre Bourdieu : Les règles de l'art; voir aussi les commentaires et applications dans Text und Feld. Bourdieu in der literaturwissenschaftlichen 
Praxis. Hg. v. Markus Joch u. Norbert Christian Wolf. Tübingen: Niemeyer 2005, et Bourdieu et la littérature. Sous la direction de Jean-Pierre Martin. Nantes: Éditions Cécile Defaut 2010.

11. Voir Jost Schneider, Sozialgeschichte des Lesens. Zur historischen Entwicklung und sozialen Differenzierung der literarischen Kommunikation in Deutschland. Berlin, New York: de Gruyter 2004, p. 202.

12. Voir, par exemple, les esquisses historiques et ethnographiques dans Der Wanderer («Die Maltheserin. Eine Episode aus den afrikanischen Kriegen », «Die Menschenjagd. Ein Bild aus dem Leben der Rothäute ») et les romans d'aventure historiques d'Eduard Breier dans le Morgen-Post (Pandur und Freimaurer, Die Sabbathianer).

13. Voir les extraits de carnets dans Ost-Deutsche Post (« Aus dem Tagebuch eines Verbannten im Kaukasus ») ou le roman poétique et féerique de Kürnberger Das Goldmärchen dans Die Donau et le roman de Betty von Ortwein Die Wiener Kartenaufschlägerin und die Modistin dans Wiener Kourier.

14. Voir les traductions de «Zwei Trauungen. Eine phantastische Erzählung» de Joseph Korzeniowski et - encore une fois - les romans d'Eduard Breier prépubliés dans le Morgen-Post.

15. Voir par exemple, "Schamyl. Frei nach Alexander Puschkin" dans Die Donau et Der letzte Fiaker. Ein Roman aus dem Wiener Volksleben d'Anton Langer ou Ein Doppelmord in Wien d'Adolph Bäuerle dans le Wiener Stadt- und Vorstadt-Zeitung.

16. Voir Pascale Casanova, La République mondiale des lettres. Paris: Seuil 1999 et Pascale Casanova: «Consécration et accumulation de capital littéraire. La traduction comme échange inégal. » In: Traduction: Les échanges littéraires internationaux. (Actes de la recherche en sciences sociales, 144, septembre 2002, numéro coordonné par Johan Heilbron et Gisèle Sapiro). Paris: Seuil 2002, p. 7-20.

17. Hubert Lengauer, «Nachwort». In: Ferdinand Kürnberger: Der Amerikamüde. Wien, Köln, Graz: H. Böhlaus Nachf. 1985, p. 568.

18. Ferdinand Kürnberger, Das Goldmärchen. Pesth, Wien und Leipzig: Hartleben 1857.

19. Ibid., p. IV-V.

20. Voir Schneider, Sozialgeschichte des Lesens, p. 252-253.

21. Lengauer: « Nachwort », p. 572.

22. Bruckmüller, Sozialgeschichte Österreichs, p. 389.

23. Rudolf Fröhlich, Die gefährlichen Klassen Wiens. Darstellung ihres Entstehens, ihrer Verbindungen, ihrer Taktik, ihrer Sitten und Gewohnheiten und ihrer Sprache. Wien: Wenedikt 1851, p. 8-9; voir aussi p. 27-28, sur « l'honneur » qui est important pour trouver du travail. Fröhlich estime qu'un quart des travailleurs viennois sont en danger de devenir criminels.

\section{ABSTRACTS}

La presse est sans doute un catalyseur et un moyen de distribution très important de la littérature populaire. Dans le cas de la monarchie habsbourgeoise le développement et l'importation de la littérature populaire étaient bloqués jusqu'en 1848 par une censure assez sévère. C'est seulement à partir des années 1850 qu'une presse populaire s'établit à Vienne, notamment le Morgenpost (Le Courrier du matin) et le Vorstadtzeitung (Le journal des faubourgs). Ces journaux incluent un roman-feuilleton avec des romans hétéronomes et locaux, d'aventure ou à thèse sociale qui se distingue nettement de celui des journaux de «qualité " avec une littérature internationale et autonome (d'après la terminologie de Pierre Bourdieu). Les lecteurs 
de ces deux groupes de journaux, eux aussi, se distinguent : une corrélation des chiffres du tirage de la presse et de la stratification sociale de la population viennoise montre que les proportions entre journaux de « qualité » et journaux populaires sont à peu près les mêmes que celles entre la bourgeoisie et l'aristocratie d'un côté et la petite bourgeoisie et les couches inférieures de l'autre. Deux exemples, le conte de fée Das Goldmärchen de Ferdinand Kürnberger et le roman populaire viennois Die Wiener Kartenschlägerin und die Modistin de Betty Ortwein von Molitor, illustrent la différence de sujet et d'écriture dans ces deux domaines littéraires.

\section{INDEX}

Mots-clés: presse, roman-feuilleton, Vienne, 1855, Ferdinand Kürnberger, Betty Ortwein von Molitor

\section{AUTHOR}

\section{NORBERT BACHLEITNER}

Norbert Bachleitner est professeur de littérature comparée à l'université de Vienne (Autriche). Ses domaines de recherche comprennent les études de réception, la traductologie, la poésie digitale, le roman au dix-neuvième siècle, la littérature et la presse, l'histoire du livre, et notamment la censure. Il a récemment publié :

- dir., avec Tone Smolej et Karl Zieger, Zola en Europe centrale. Valenciennes: Presses universitaires de Valenciennes 2011;

Fiktive Nachrichten. Die Anfänge des europäischen Feuilletonromans. Würzburg: Königshausen \& Neumann 2012;

dir., avec Murray G. Hall, „Die Bienen fremder Literaturen“. Der literarische Transfer zwischen Großbritannien, Frankreich und dem deutschsprachigen Raum im Zeitalter der Weltliteratur (1770-1850).

Wiesbaden: Harrassowitz 2012;

- dir., avec Christine Ivanovic, Nach Wien! Sehnsucht, Distanzierung, Suche. Literarische Darstellungen Wiens aus komparatistischer Perspektive. Frankfurt/Main: Lang 2015;

- Die literarische Zensur in Österreich von 1751 bis 1848. Wien, Köln, Weimar: Böhlau 2017.

Pour plus de détails voir http://complit.univie.ac.at/die-abteilung/wissenschaftlichemitarbeiterinnen/bachleitner-norbert/?no_cache=1. 